\title{
Identification of a New Form of AQP4 mRNA That Is Developmentally Expressed in Mouse Brain
}

\author{
SERGEY ZELENIN, ELI GUNNARSON, TATYANA ALIKINA, ALEXANDER BONDAR, AND \\ ANITA APERIA
}

Department of Woman and Child Health, Karolinska Institutet, Astrid Lindgren Children's Hospital, 171

76 Stockholm, Sweden [E.G., A.A.]; and Novosibirsk Institute of Bioorganic Chemistry, Siberian Branch of the Russian Academy of Sciences, 630090, Novosibirsk, Russia [S.Z., T.A., A.B.]

\section{ABSTRACT}

The water channel aquaporin 4 (AQP4) is abundantly expressed in the brain, and also in lung and kidney. Previous studies have suggested that there are at least two AQP4 mRNA. The two mRNA encode for two AQP4 proteins that differ with regard to the length of the N-terminal: AQP4.M1 and AQP4.M23. Here we report, by use of reverse transcriptase PCR and comparison of genomic and cDNA structures, the presence of a third form of mouse AQP4 mRNA. The upstream sequence of this form of mRNA originates from an additional exon, interspaced between exon 0 and exon 1, and an alternatively spliced form of exon 1. Analysis of nucleotide sequence suggests that this new form of AQP4 mRNA also encodes for the AQP4.M23 protein. The two forms of AQP4 mRNA that presumably both encode for M23 have a tissue- and age-specific expression. The new AQP4 mRNA was predominantly expressed in brain. The expression was approximately twofold higher in the adult brain than in the infant brain. In contrast, the expression levels of the new mRNA were low in both infant and adult lung and kidney. The previously described mRNA encoding for AQP4.M23 was predominantly expressed in lung and kidney. In lung, the expression of this form was higher in infancy than in adulthood. In conclusion, we have identified a new form of AQP4 mRNA that is predominantly expressed in the brain and that is developmentally regulated. (Pediatr Res 48: 335-339, 2000)

AQP4, aquaporin 4

Abbreviations

RT-PCR, reverse transcriptase PCR

MIWC, mercurial-insensitive water channel
Maintenance of brain water homeostasis is of utmost importance. Diffuse brain swelling often occurs as a consequence of trauma, and this complication is particularly common in children (1). The mechanisms regulating brain water homeostasis are, despite the enormous importance of the subject, incompletely known. There is also little information about whether this regulation is age dependent.

It has been suggested that AQP4, a water channel that is expressed in the brain, plays a role in the regulation of brain water homeostasis. AQP4 is localized in the astrocytes and ependymal cells. It is abundantly expressed in membranes facing blood vessels and the subarachnoidal space (2). This has been taken as indirect evidence of its role in water exchange between brain and blood and cerebrospinal fluid. There are at

Received January 4, 2000; accepted April 4, 2000.

Correspondence and reprint requests: Dr. Anita Aperia, Department of Woman and Child Health, Karolinska Institutet, Astrid Lindgren Children's Hospital, Q2:09, 17176 Stockholm, Sweden.

This work was supported in part by the Swedish Medical Research Council (03644), the Märta and Gunnar V. Philipson Foundation, INTAS grant no. INTAS-97-11404, and by the RFBR grant no. 98-04-49369. least two isoforms of the AQP4 protein-M1 and M23. The M1 form contains 22 more amino acids in the N-terminal than the M23 form. The M23 isoform has been reported to be encoded by four exon segments (3). The mRNA encoding the M1 protein has been reported to contain an additional nucleotide sequence corresponding to an exon segment (exon 0), upstream of exon 1 in the mouse AQP4 gene. There are, however, conflicting data $(3,4)$ concerning the mRNA sequence of AQP4 and the number of distinct AQP4 mRNA isoforms. This has prompted us to reexamine the expression of AQP4 mRNA in mouse tissue. Three forms were identified, which we will name AQP4.M1 (encoding for M1), AQP4.M23X (a new form of AQP4 mRNA), and AQP4.M23 (the form previously described to encode for M23). In each case, the AQP4 mRNA sequence was confirmed by comparison with sequence of genomic DNA.

We studied the age and tissue dependence of the expression of both forms of AQP4 mRNA-AQP4.M23X and AQP4.M23 - that presumably encode the M23 protein. The AQP4.M23X mRNA form was found to be expressed at relatively high levels in the adult brain and at low levels in the lung 
and kidney. In contrast, the AQP4.M23 mRNA form was detected at relatively low levels in the brain compared with lung and kidney. The expression of the AQP4.M23X mRNA form in the brain was found to be age dependent. This was not the case for the AQP4.M23 mRNA form.

\section{MATERIALS AND METHODS}

Structure analysis of AQP4 gene and AQP4 mRNA. A 1.6-kb PCR fragment was prepared by amplification of mouse genomic DNA (Promega, Madison, WI, U.S.A.) using primers corresponding to exon 1 (sense) and 2 (antisense) of the AQP4 gene. This fragment was labeled with $\alpha$ - $\mathrm{P}^{32}$ deoxycytidine triphosphate (random priming; Amersham Life Science, Little Chalfont, Buckinghamshire, U.K.) and used for screening of the mouse genomic library. The $\lambda$ DNA sample of the clone containing AQP4 gene was prepared, digested with $X b a \mathrm{I}$ restriction enzyme or NotI and BamHI, and subcloned into plasmid pBluescript II KS+ (Stratagene, La Jolla, CA, U.S.A.). Clones were analyzed for presence of the $5^{\prime}$ end AQP4 gene by Southern hybridization with oligonucleotides corresponding to the $5^{\prime}$ end of the AQP4 mRNA. Using the pBluescript Exo/Mung DNA Sequencing System (Stratagene) protocol, we obtained gene fragments with predictable size of deletion. Sequencing was performed using the Sequenase Version 2.0 DNA Sequencing Kit (Amersham) and the Thermo Sequenase Dye Terminator Cycle Sequencing Pre-Mix Kit (Amersham).

Total RNA was extracted from adult mouse brain using RNeasy Total RNA Kit (QIAGEN, Hilden, Germany). Reverse transcription and PCR were, with few modifications, carried out as described (5). PCR fragments were isolated by electrophoresis, extracted using QIAquick Gel Extraction Kit (QIAGEN), and used directly for sequencing by the dideoxy chain termination method (ABI Prism Dye Terminator Cycle Sequencing Ready Reaction Kit; PE Applied Biosystems, Foster City, CA, U.S.A.).

AQP4.M23X mRNA was quantified by semiquantitative RT-PCR using the following primers: sense AQ4.CF1, 5'TTATGGTTCACGGGTTTGGATG-3'; antisense AQP4.460, 5'-TGGTGACTCCCAATCCTCCAAC-3'; size of fragment $749 \mathrm{bp}$; or antisense AQP4.078, 5' - AAAGCCACCATGATGCTC-3'; size of fragment 367 bp. AQP4.M23 mRNA was quantified using the following primers for exon 1: sense AQP4.001, 5'-GGAAGGCTAGGTTGGTGACTTC-3' and antisense AQP4.460, 5'-TGGTGACTCCCAATCCTCCAAC$3^{\prime}$; size of fragment $460 \mathrm{bp}$. $\beta$-Actin was amplified as internal control using the following primers: sense $\beta$-Act.518, 5' TGGTCGTACCACAGGCATTGTG-3'; antisense $\beta$-Act.1202, 5'-AAGGGTGTAAAACGCAGCTCAG-3', size of fragment 726. All primers were designed based on their reported sequences with use of MacVector 4.1.4. (Kodak, New Haven, CT, U.S.A.) $(3,6)$.

The following criteria for PCR primer design were used: size of all primers was 18-22 nucleotides, $\mathrm{G} \& \mathrm{C}$ nucleotide content no more than $55 \%$, and melting temperature was in the range of $56-60^{\circ} \mathrm{C}$. All primers were analyzed against GeneBank sequence data for absent high homology to some alien nucleotide structure.
Semiquantitative analysis of AQP4 mRNA expression. The studies were performed on adult and infant mice of the C57 Black strain. The infant mice were killed on the second postnatal day ( $2 \mathrm{~d}$ old). The adult mice were allowed free access to food and water. The infant mice were kept with their dams. All animals were killed by decapitation. Brain, lung, and kidney tissues were removed and directly frozen on $\mathrm{CO}_{2}$ ice and kept at $-80^{\circ} \mathrm{C}$ until analysis.

Total RNA was extracted from brain, kidney, and lung tissues of 2-d-old and adult mice by using an RNeasy Total RNA Kit (QIAGEN). Reverse transcription and PCR were, with few modifications, carried out as described (5). All samples used for quantification were cycled in linear conditions at 28 and 30 cycles at least two times with three different RNA preparations. All reaction solutions (except RNA) in the semiquantitative RT-PCR were premixed to eliminate errors during pipetting.

To measure PCR product amount, $5 \mu \mathrm{Ci}$ of $\alpha$-P (32) deoxycytidine triphosphate in $100 \mu \mathrm{L}$ of PCR mixture were used; 10 $\mu \mathrm{L}$ of PCR mixture were separated on a $4 \%$ polyacrylamide gel (PAAG) and stained with $0.5 \mu \mathrm{g} / \mathrm{mL}$ ethidium bromide. $\beta$-Actin and AQP4 fragments and the space between them of corresponding size were cut from the gel under UV light and analyzed on a $\beta$-counter.

The animal study has been approved by the ethical committee of the Karolinska Institute.

\section{RESULTS}

Mouse AQP4 gene cloning, mapping, and sequence analysis. Screening of a mouse genomic library with a 1.6-kb PCR fragment, corresponding to the intron between exon 1 and 2 of mouse AQP4 gene, yielded five independent clones. Southern analysis indicated a single copy of the AQP4 gene in mouse genome. Restriction mapping revealed two clones that covered approximately a 27-kb genomic fragment and included $9 \mathrm{~kb}$ upstream of the $5^{\prime}$ end of exon 0 and $7 \mathrm{~kb}$ downstream of the $3^{\prime}$ end of exon 4. Fragments, corresponding to the $5^{\prime}$ end flanking region of the AQP4, were subcloned for restriction mapping and sequence analysis. Comparison of genomic DNA sequence of this region and AQP4 cDNA sequence data showed the presence of an exon that was interspaced between exon 0 and exon 1 (Fig. 1). In this article we will refer to this exon as "exon X."

AQP4 mRNA structure analysis. By use of a set of primers, we identified three AQP4 mRNA, which will be named AQP4.M1 (encoding for M1), AQP4.M23X, a new form, and AQP4.M23 mRNA (Fig. 2).

The sequence of AQP4.M1 mRNA form (Fig. 3a) was similar to the sequence of the AQP4 M1 mRNA reported by Turtzo et al. (4) and MIWC2 reported by Ma et al. (3). However, in contrast to Turtzo et al., we found 105 additional nucleotides at the $5^{\prime}$ end of the exon 0 . We did not confirm the presence of the first 32 nucleotides at the $5^{\prime}$ end of the AQP4 M1 structure that this group has reported. These 32 nucleotides are not present in structure of the genomic fragment. 

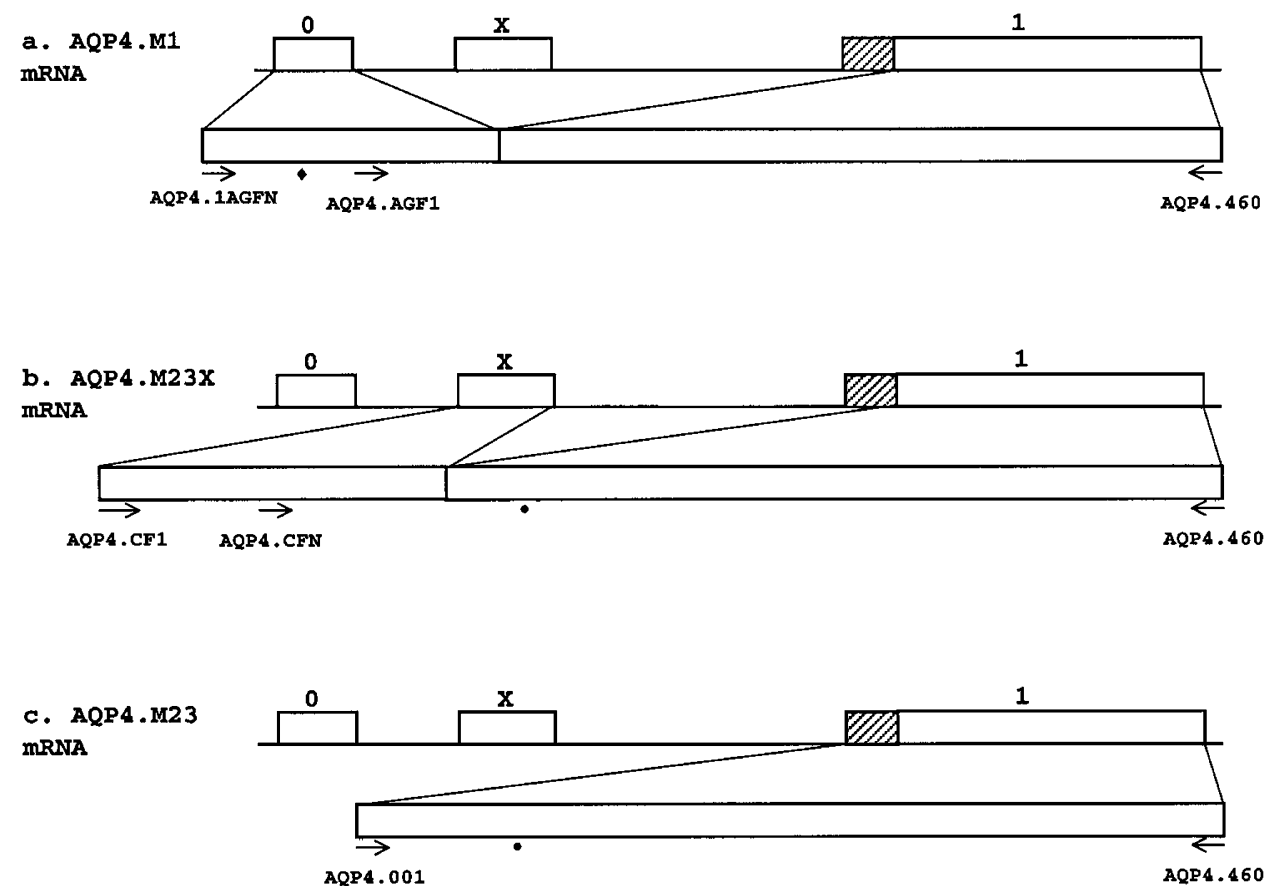

Figure 1. Scheme of the mouse AQP4 gene fragment, based on the nucleotide sequence of the genomic and cDNA fragments. (a-c) Formation of alternative AQP4 mRNA forms. The squares indicate exon $(0, X, 1)$ segments and corresponding mRNA structures. The dashed box represents part of exon 1 that is spliced out in AQP4.M1 mRNA and AQP4.M23X mRNA. Arrows show position of PCR primers. Position of ATG codon starting the M1 and M23 proteins are shown as $(\diamond)$ and $(\bullet)$, respectively.

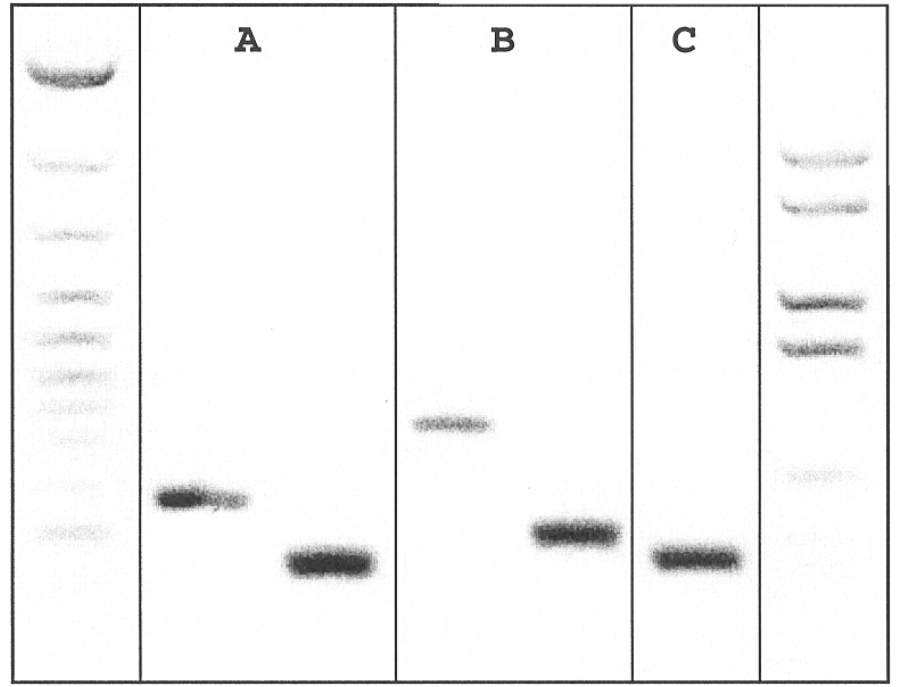

Figure 2. Detection of AQP4 mRNA isoforms by RT-PCR. The cDNA sample, corresponding mouse brain RNA, was used as a template for PCR amplifications using the identical antisense primer AQP4.460 and a number of sense primers. For details regarding positions of PCR primers see Figure 1. $(A)$ PCR fragments, produced by using AQP4.1AGFN or AQP4.AGF1 oligonucleotides as sense primers, corresponding to exon 0. (B) PCR fragments, produced by using AQP4.CF1 or AQP4.CFN oligonucleotides as sense primers, corresponding to nucleotide sequence interspaced between exon 0 and exon 1. (C) PCR fragment, produced by using AQP4.001 oligonucleotide as sense primer, corresponding to exon 1.

A second form, AQP4.M23X mRNA (Fig. 3b) was similar to the AQP4 mRNA mMIWC3 described by Ma et al. (3). Comparison of genomic and cDNA structures did, however, reveal that AQP4.M23X mRNA includes a sequence that corresponds to an exon that is interspaced between exon 0 and exon 1 of the mouse
AQP4 gene. Within this sequence we identified 206 additional nucleotides. Our analysis also indicated that alternative splicing of exon 1 had occurred. Analysis of the AQP4.M23X mRNA open reading frames indicated that the first optimal context for initiation of translation (7) was localized at the site for start of translation of the AQP4 M23 protein.

A third form of AQP4 mRNA, AQP4.M23 mRNA (Fig. 3c), corresponded to the MIWC1 mRNA reported by Ma et al. (3).

Tissue distribution of the AQP4.M23X and AQP4.M23 $\boldsymbol{m} \boldsymbol{R} \boldsymbol{N} \boldsymbol{A}$. The two AQP4 mRNA that presumably encode for the same AQP4 M23 protein, were analyzed with regard to tissue and developmental expression using semiquantitative RTPCR. We examined tissues from brain, lung, and kidney in 2-d-old and adult mice. These tissues have previously been reported to express AQP4 (3). A $\beta$-actin control was included in the PCR as an internal standard. All expression levels of AQP4 mRNA were related to the expression of the $\beta$-actin. Representative PCR on agarose gel for the AQP4.M23X mRNA and AQP4.M23 mRNA forms are shown in Figure $4 a$ and $4 b$, respectively. The relative expression of the two different AQP4 mRNA forms (related to $\beta$-actin transcript) in the adult brain was arbitrarily assigned a value of unity for comparison with the other tissues and ages.

In tissue from adult mice the expression of the AQP4.M23X mRNA was, in relative terms, highest in the brain (Fig. 4c). In contrast, only low levels of AQP4.M23X mRNA were detected in the lung and kidney compared with the brain (Fig. 4c). AQP4.M23 mRNA was less abundantly expressed in the adult brain than in lung and kidney (Fig. 4d).

The AQP4.M23X mRNA had a significantly lower expression in infant mouse brain than in the adult brain (Fig. 4c). The 


\section{a}

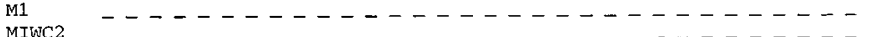
Genomic...ct tactgattGCCACATGGTGCAGAATCTTTCCACCCCTACTCTCCAAAAACCCAATCAGACA M1 _. . . . . . . GACCCAAGCTTAGATCTGGCTPTCAAAGGCGTCTGGACTCAAG MIWC2
AQP4 M1 AGTGCCCGTAATCTGACTCCCAGTGTACTGGAGCCCGGGGGCAGGCACTGAGCTGCACTCTGG Genomic AGTGCCCGTAATCTGACTCCCAGTGTACTGGAGCCCGGGGGCAGGCACTGAGCTGCACTCTGG Genomic AGTGCCCGTAATCTGACTCCCAGTGTACTGGAGCCCGGGGGCAGGC $\begin{array}{ll}\text { M1 } & \text { CCAGGGAAGGCATGAGTGACGGAGCTGCAGCGAGGCGGTGGGG... } \\ \text { MIWC2 } & \text {.-GAGGGAAGG_ATTAGTGACGGAGCTGCAGCGAGGCGTGGGG.. }\end{array}$ $\begin{array}{lll}\text { MIWC2 } & \text { - GAGGGAAGG_ATGAGTGACGGAGCTGCAGCGAGGCGGTGGGG... } \\ \text { AQP4 .M1 CCAGGGAAGGOATGAGTGACAGAGCTGCGGCAAGGCGTGGG... }\end{array}$ Genomic CCAGGGAAGGQATGAGTGACAGAGCTGCGGCAAGGCGGTGGGGgtaagtcct. Met 1

\section{b}

MIWC3

$5^{\prime}-$ TTATGGT'TCACGGGTTTGGATGCTTTGTTT
Genomic.. T2

5 ' -AAAGTCATTTTAAAGTTTCTATTTATGGTTCACGGGTTTGGATGCTTTGTTT

$\begin{array}{ll}\text { MIWC3 } & \text { TTTTCTTTPTAAT } \\ \text { AOP4.M23X TTTTCTTTTAATTTCTCTGATGAAAAATGAGGTTCAAGAGCAACTGAGTTTCTTGATTA }\end{array}$

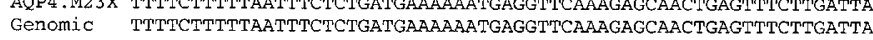

MIWC3

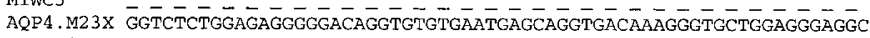

Genomic GGTCTCTGGAGAGGGGGACAGGTGTGTGAATGAGCAGGTGACAAAGGGTGCTGGAGGGAGGC

MIWC3

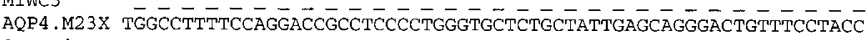

Genomic TGGCCTTTTCCAGGACCGCCTCCCCTGGGTGCTCTGCTATTGAGCAGGGACTGTTTCCTACC

MIWC3
AQP4.M23X CACCCTGCTCCCTGCACTTCCTGCTTGGTCCCCTTCTCTTTCCTCTCTCTGGGCCTCAGAAG

Genomic CACCCTGCTCCCTGCACTTCCTGCTTGGTCCCCTTCTCTTTCCTCTCTCTGGGCCTCAGAAG

MIWC 3 ACAGCACCTGTAATAGCACTTTGCCCCTCTGCCACCTGGCTACAACCCTGGACTGCTG... AQP4.M23X ACAGCACCTGTAATAGCACTTTGCCCCTCTGCCACCTGGCTACAACCCTGGACTGCTG

Genomic ACAGCACCTGTAATAGCACTTTGCCCCTCTGCCACCTGGCTACAACCCTGGACTGCTGgtg... c

M1 MIWC2 AQP4.M $\mathrm{AQP} 4 . \mathrm{M} 23 \mathrm{x}$ Genomic... M1 MIWC2 $\mathrm{AQP4} . \mathrm{M} 1$ MIWC3 Genomic GTCTGGACTCAGGCTTTCTGGAAGGCAGTCTCAGCAGAATTTCTGGCCACACTTATCTTTGTT M1 TTGCTCGGTGTGGATCCACCATAAACTGGGGTGCTCAGAAAACCCCTTACCTGTGGACATG MIWC2 TIC

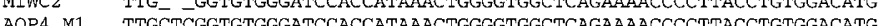
AQP4.M1

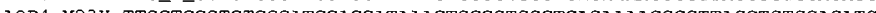
Genomic TrGCTCGGTGTGGGATCCACCATAAACTGGGGTGGCTCAGAAAACCCCTTACCTGTGGACATG

M1 MIWC2 GICCTCATCTCCCTTTGCTTTGGACTCAGCATTGCTACCATGGTGCAGTGCTTTGGCCACATC AQP4 GTCCTCATCTCCCTTTTCTTTGGACTCAGCATTGCAACCATGGTGCAGTGCCTTGGCCACATC MIWC3 GICCTCATCTCCCTTTGCTTTGGACTCAGCATTGCTACCATGGTGCAGTGCTTTGGCCACATC GTCCTCATCTCCCTRTGCTTTGGACTCAGCATTGCAACCATGGTGCAGTGCCTTGGCCACATC AQP4.M23X GTCCTCATCTCCCTTTGCTTTGGACTCAGCATTGCTACCATGGTGCAGTGCTTTGGCCACATC
Genomic GTCCTCATCTCCCTTTCTTTGGACTCAGCATTGCTACCATGGTGCAGTGCTTTGGCCACATC

M1 AGTGGTGGCCACATCAATCCCGCTGTGACTGTAGCCATGGTGTGCACACGAAAGATCAGCATC MIWC2 AGCGGTGGCCACATCAATCCAGCTGTGACTGTAGCCATGGTGTGCACACGAAAGATCAGCATC AQP4.M1 AGTGGTGGCCACATCAATCCCGCTGTGACTGTAGCCATGGTGTGCACACGAAAGATCAGCATC MIWC3 AGCGGTGGCCACATCAATCCAGCTGTGACTGTAGCCATGGTGTGCACACGAAAGATCAGCATC AQP4.M23X AGTGGTGGCCACATCAATCCCGCTGTGACTGTAGCCATGGTGTGCACACGAAAGATCAGCATC AQP4.M23X AGTGGTGGCCACATCAATCCCGCTGTGACTGTAGCCATGGTGTGCACACGAAAGATCAGCATC
Genomic

M1 GCTAAGTCCGTCTTCTACATCATTGCACAGTGCCTGGGGGCCATCATTGGAGCCGGCATCCTC MIWC2 GCTAAGTCCGTCTTCTACATCATTGCACAGTGCCTGGGGGCCATCATTGGAGCCGGCATCCTC AQP4.M1 GCTAAGTCCGTCTTCTACATCATTGCACAGTGCCTGGGGGCCATCATTGGAGCCGGCATCCTC MIWC3 GCTAAGTCCGTCTTCTACATCATTGCACAGTGCCTGGGGGCCATCATTGGAGCCGGCATCCTC AQP4.M23X GCTAAGTCCGTCTTCTACATCATTGCACAGTGCCTGGGGGCCATCATTGGAGCCGGCATCCTC Genomic GCTAAGTCCGTCTTCTACATCATTGCACAGTGCCTGGGGGCCATCATTGGAGCCGGCATCCTC

M1 TACCTGGTCACACCTCCCAGTGTGGTTGGAGGATTGGGAGTCACCACG... MIWC2 TACCTGGTCACACCTCCCAGTGTGGTTGGAGGATTGGGAGTCACCACG... AQP4.M1 TACCTGGTCACACCTCCCAGTGTGGTTGGAGGATTGGGAGTCACCACG... MIWC3 TACCTGGTCACACCTCCCAGTGTGGTTGGAGGATTGGGAGTCACCACG... AQP4.M23X TACCTGGTCACACCTCCCAGTGTGGTTGGAGGATTGGGAGTCACCACG..

Genomic TACCTGGTCACACCTCCCAGTGTGGTTGGAGGATTGGGAGTCACCACGgtgagaactctt...

Figure 3. Partial nucleotide structures of the mouse AQP4 gene (exon $0, \mathrm{X}$, and part of exon 1) and related AQP4 RNA fragments in relation to previously published sequence for mouse AQP4 mRNA. M1 mRNA reported by Turtzo et al. (4) and MIWC2, MIWC3 reported by Ma et al. (3). Absent nucleotides in AQP4.M1, MIWC2, and MIWC3 structures are marked as “ ". Dissimilar nucleotides in AQP4.M1, MIWC2, and MIWC3 structures are presented in bold. Positions of ATG codon starting AQP4.M1 and AQP4.M23 proteins are boxed. Nucleotides of the exons and related AQP4 mRNA fragments are presented by capital letters and flanking AQP4 genomic structure by lowercase letters. (a) Nucleotides of the exon 0 and related AQP4.M1, MIWC2, and M1 mRNA. (b) Nucleotide structure of the mouse AQP4 gene region, including the exon, that is interspaced between exon 0 and exon 1 and related AQP4.M23X and MIWC3 mRNA nucleotide sequences. (c) Nucleotides of the exon 1 fragment and related AQP4 mRNA fragments.

relative expression of the AQP4.M23 mRNA was very low in both infant and adult brain (Fig. 4d). In the lung, the relative expression of the AQP4.M23 mRNA was significantly higher in the infant than the adult (Fig. 4d). This finding is consistent with the developmental pattern of AQP4 previously reported from the rat lung (8). The AQP4.M23X mRNA was expressed at low levels in both infant and adult lung and kidney (Fig. 4c).

\section{DISCUSSION}

This study demonstrates that the mouse AQP4 gene can give rise to at least three different mRNA, and that two of those, AQP4.M23X mRNA and AQP4.M23 mRNA, presumably encode for the same protein, i.e. AQP4 M23. We report an AQP4 exon that has not been previously described. The AQP4.M23X mRNA was found to have a sequence corresponding to this exon.

Several discrepancies were found with regard to previously reported forms of AQP4 mRNA. In this study, the sequence data of the AQP4 mRNA were confirmed by comparison with the mouse AQP4 gene sequence. We suggest that the differences in AQP4 mRNA structures between present and previous studies might be the consequences of artifacts during cDNA library construction. It is known that artifacts of cDNA cloning may occur because of complex secondary structure of some mRNA $(9,10)$.

Of great interest was the finding that the two forms of AQP4 mRNA, which presumably encode the same protein, have both a tissue- and age-specific expression. The AQP4.M23X mRNA was more abundantly expressed in brain than in the peripheral tissues examined, and might represent a brain-specific form of AQP4 mRNA of the M23 protein. As a consequence the regulation of AQP4 M23, protein expression may be different in brain and in peripheral tissue. The two mRNA may vary with regard to transcription of the gene, RNA stability, and/or translation of the protein. The biologic significance of this needs to be taken into account in future studies. Interestingly, there are now several reports suggesting that the $5^{\prime}$ untranslated region $\left(5^{\prime}\right.$-UTR) is important for the regulation of mRNA stability $(11,12)$.

There was a pronounced postnatal increase in the expression of the AQP4.M23X mRNA form in the brain. It will be an important topic for the future studies to examine the relative expression of AQP4 M23X versus AQP4 M23 mRNA in distinct regions of the brain. A recent publication (13) has 
a

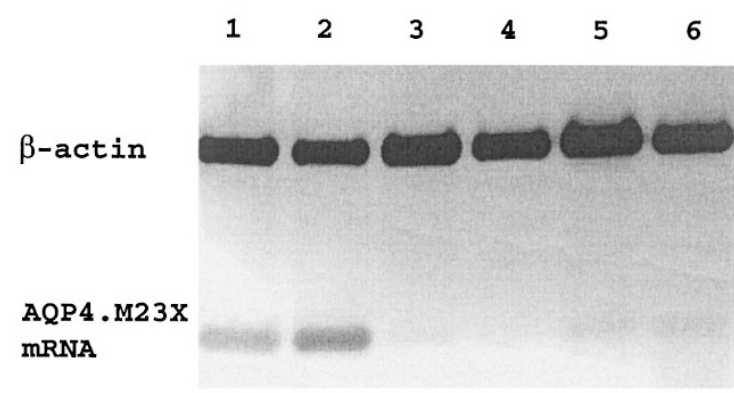

b

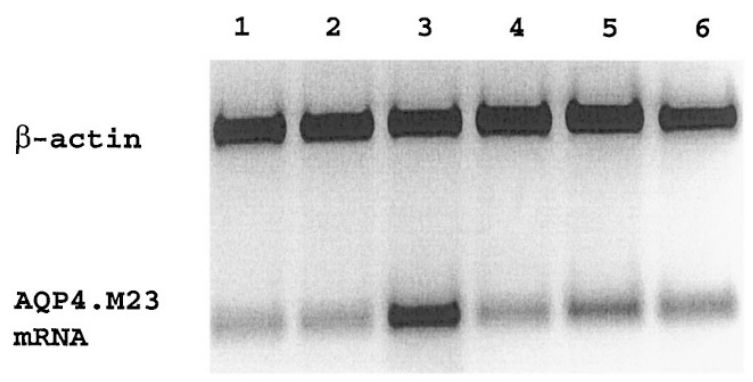

C

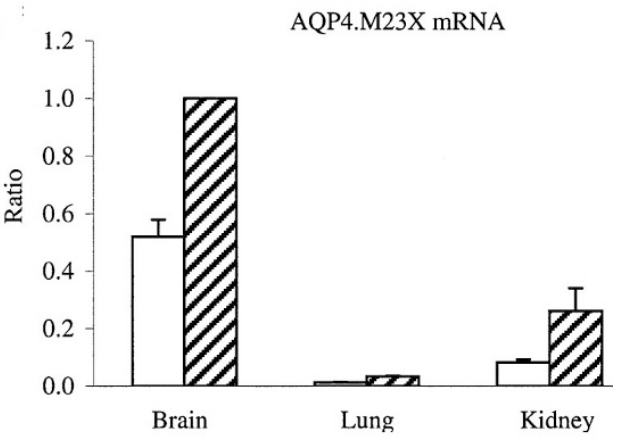

d

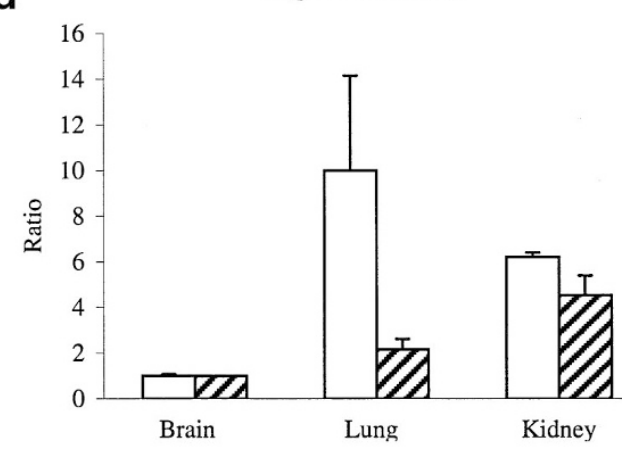

Figure 4. Representative RT-PCR and relative ratios of AQP4.M23X and AQP4.M23 mRNA expressions. (a) Representative RT-PCR on agarose gel showing bands corresponding to the internal standard $\beta$-actin and the AQP4.M23X mRNA in 2-d-old and adult mouse brain (lanes 1 and 2), lung (lanes 3 and 4), and kidney (lanes 5 and 6 ), respectively. (b) Representative RT-PCR on agarose gel showing bands corresponding to the internal standard $\beta$-actin and the AQP4.M23 mRNA in 2-d-old and adult mouse brain (lanes 1 and 2), lung (lanes 3 and 4), and kidney (lanes 5 and 6), respectively. (c) Ratios based on calculations from semiquantitative RT-PCR of the AQP4.M23X mRNA abundance in 2-d-old (open bars) and adult (hatched bars) mouse brain, lung, and kidney. The relative expression of the AQP4.M23X mRNA transcript (related to the $\beta$-actin transcript) in the adult brain was arbitrarily assigned a value of unity for comparison with the other tissues and ages. Each bar represents the mean \pm SEM of minimum samples from different animals. $(d)$ Ratios based on calculations from semiquantitative RT-PCR of the AQP4.M23 mRNA abundance in 2-d-old (open bars) and adult (hatched bars) mouse brain, lung, and kidney. The relative expression of the AQP4.M23 mRNA transcript (related to the $\beta$-actin transcript) in the adult brain was arbitrarily assigned a value of unity for comparison with the other tissues and ages. Each bar represents the mean \pm SEM of three tissue samples from different animals.

added evidence to the hypothesis of AQP4 playing a key role in conditions of brain edema. It is well established that infants and children are more susceptible than adults to develop diffuse and rapid cerebral swelling after, for instance, traumatic brain injury $(1,14)$. This indicates that regulation of brain water homeostasis is not yet fully developed in childhood. Our finding of a low expression of a brain-specific AQP4 mRNA may be relevant for the understanding of how regulation of brain water homeostasis is enhanced postnatally.

Acknowledgment. The authors thank Lill-Britt Svensson for technical assistance.

\section{REFERENCES}

1. Aicardi J, Bax M, Gillberg C, Ogier H 1998 Disease of the Nervous System in Childhood, 2nd Ed. Cambridge University Press, London, pp 460-488

2. Nielsen S, Nagelhus EA, Amiry-Moghaddam M, Bourque C, Agre P, Ottersen OP 1997 Specialized membrane domains for water transport in glial cells: high-resolution immunogold cytochemistry of aquaporin-4 in rat brain. J Neurosci 17:171-180

3. Ma T, Yang B, Verkman AS 1996 Gene structure, cDNA cloning and expression of a mouse mercurial-insensitive water channel. Genomics 33:382-388

4. Turtzo LC, Lee MD, Lu M, Smith BL, Copeland NG, Gilbert DJ, Jenkins NA, Agre P 1997 Cloning and chromosomal localization of mouse aquaporin 4: exclusion of a candidate mutant phenotype, ataxia. Genomics 41:267-270
5. Yasui M, Marples D, Belusa R, Eklof AC, Celsi G, Nielsen S, Aperia A 1996 Development of urinary concentrating capacity: role of aquaporin-2. Am J Physiol 271:F461-F468

6. Sadano H, Taniguchi S, Kakunaga T, Baba T 1988 cDNA cloning and sequence of a new type of actin in mouse B16 melanoma. J Biol Chem 263:15868-15871

7. Kozak M 1996 Interpreting cDNA sequences: some insights from studies on translation. Mamm Genome 7:563-574

8. Yasui M, Serlachius E, Löfgren M, Belusa R, Nielsen S, Aperia A 1997 Perinatal changes in expression of aquaporin-4 and other water and ion transporters in rat lung. J Physiol 505:3-11

9. Spring DJ, Krebs EG 1999 Deletion of 11 amino acids in p90(rsk-mo-1) abolishes kinase activity. Mol Cell Biol 19:317-320

10. Jha PK, Sarkar S 1997 DNA sequencing and comparative sequence analysis reveal that the Escherichia coli genomic DNA may replace the target DNA during molecular cloning: evidence for the erroneous assembly of E. coli DNA into database sequences. Comp Biochem Physiol B Biochem Mol Biol 118:333-339

11. D'erchia AM, Pesole G, Tullo A, Saccone C, Sbisa E 1999 Guinea pig p53 mRNA: identification of new elements in coding and untranslated regions and their functional and evolutionary implications. Genomics 58:50-64

12. Dibbens JA, Miller DL, Damert A, Risau W, Vadas MA, Goodall GJ 1999 Hypoxic regulation of vascular endothelial growth factor mRNA stability requires the cooperation of multiple RNA elements. Mol Biol Cell 10:907-919

13. Vizuete ML, Venero JL, Vargas C, Ilundain AA, Echevarría M, Machado A, Cano J 1999 Differential upregulation of aquaporin-4 mRNA expression in reactive astrocytes after brain injury: potential role in brain edema. Neurobiol Dis 6:245-258

14. Kochanek PM, Bell MJ, Forbes ML, Adelson PD, Clark RSB 1999 Pediatric brain Injuries: pathophysiology. In: Marion DW (ed) Traumatic Brain Injury. Thieme, New York, pp 233-256 